Article

\title{
Vibration of Rotating and Revolving Planet Rings with Discrete and Partially Distributed Stiffnesses
}

\author{
Fuchun Yang ${ }^{1,2, *(1)}$ and Dianrui Wang ${ }^{1}$ \\ 1 Key Laboratory of High Efficiency and Clean Mechanical Manufacture of MOE, School of Mechanical \\ Engineering, Shandong University, Jinan 250061, China; 201934000@mail.sdu.edu.cn \\ 2 National Demonstration Center for Experimental Mechanical Engineering Education, Shandong University, \\ Jinan 250061, China \\ * Correspondence: fuchunyang@sdu.edu.cn
}

Citation: Yang, F.; Wang, D. Vibration of Rotating and Revolving Planet Rings with Discrete and Partially Distributed Stiffnesses. Appl. Sci. 2021, 11, 127. https://dx.doi.org/ 10.3390/app11010127

Received: 12 November 2020 Accepted: 23 December 2020 Published: 24 December 2020

Publisher's Note: MDPI stays neutral with regard to jurisdictional claims in published maps and institutional affiliations.

Copyright: (c) 2020 by the authors. Licensee MDPI, Basel, Switzerland. This article is an open access article distributed under the terms and conditions of the Creative Commons Attribution (CC BY) license (https: / / creativecommons.org/ licenses/by/4.0/).

\begin{abstract}
Vibration properties of high-speed rotating and revolving planet rings with discrete and partially distributed stiffnesses were studied. The governing equations were obtained by Hamilton's principle based on a rotating frame on the ring. The governing equations were cast in matrix differential operators and discretized, using Galerkin's method. The eigenvalue problem was dealt with state space matrix, and the natural frequencies and vibration modes were computed in a wide range of rotation speed. The properties of natural frequencies and vibration modes with rotation speed were studied for free planet rings and planet rings with discrete and partially distributed stiffnesses. The influences of several parameters on the vibration properties of planet rings were also investigated. Finally, the forced responses of planet rings resulted from the excitation of rotating and revolving movement were studied. The results show that the revolving movement not only affects the free vibration of planet rings but results in excitation to the rings. Partially distributed stiffness changes the vibration modes heavily compared to the free planet ring. Each vibration mode comprises several nodal diameter components instead of a single component for a free planet ring. The distribution area and the number of partially distributed stiffnesses mainly affect the high-order frequencies. The forced responses caused by revolving movement are nonlinear and vary with a quasi-period of rotating speed, and the responses in the regions supported by partially distributed stiffnesses are suppressed.
\end{abstract}

Keywords: vibration; planet ring; revolving movement; partially distributed stiffness

\section{Introduction}

Vibration analysis of rotation rings in different structures, such as gears, rotors, bearings, etc., is of great interest for complex dynamic characteristics. This study was motivated by planetary gear systems, where planet gears often have a thin rim [1], especially in aerospace applications, where weight is an important consideration. Planet gears do not only rotate around their axis supported by bearings but revolve around the center of planetary gear systems meshed with central gears. Meanwhile, they are supported by both discrete stiffnesses of mesh pairs and partially distributed stiffnesses of support bearings. These planet gears may undergo significant elastic continuum vibration during operation, for their compliance, combined movement, and complex elastic supports.

Compliance of gears has received attention in some references. Talbert and Gockel [2] found elastic vibration in aerospace gears experimentally. Bettaieb et al. [3] investigated the flexible gear vibration, using FEM method. Elastic deformations are also found in planetary gears by experiments [4]. Vinayak and Singh [5] studied the vibration modes of compliant gears by multibody dynamic formulation. Kahraman et al. [6] investigated the effect of flexibility of gears on the dynamic behavior of a planetary gear set. Their study shows that dynamic gear tooth bending stress values were found to be significantly higher for all of the gears for a flexible internal gear as compared to the rigid internal gear. 
Vibrations of stationary or rotating rings with different types of supports have been investigated by many researchers. Bert and Chen [7] studied the in-plane and out-plane vibration of rotating rings on an elastic foundation. Free vibrations of rings on rigid radial supports were investigated by Rao and Sundararajan [8], where the ring was separated into several parts with supports located at the ends of each segment. Detinko [9] studied the free vibration of a thick ring on multiple radial supports by the Galerkin method. Bickford and Reddy [10] investigated rotating rings, where discrete stiffnesses are attached to and rotate with the ring. Allaei et al. [11] investigated the vibration of non-axisymmetric rings with lumped masses and stiffnesses added. Loy and Lam [12] studied the cylindrical shell with ring support. Metrikine and Tochilin [13] studied the steady state response of an elastic ring subjected to a uniformly moving load by the method of images. The results show that, for small velocities of the load, the ring pattern is almost perfectly symmetric with respect to the loading point. The pattern becomes slightly asymmetric due to the viscosity of the springs if the load velocity is small. Kim and Chung [14] investigated the free non-linear vibration of a rotating thin ring with in-plane and out-plane motions. Nackenhorst and Brinkmeier [15] used two observer systems, that is, Lagrangian and Eulerian systems, to derive rotating ring models. Wu and Parker [16] studied the vibration of rings on a general elastic foundation. There are fewer studies on the vibration of rotating rings with discrete supports. Afterward, they [17] studied the modal properties of equally spaced planetary gears with elastic ring gears. The results show that all eigenfunctions fall into four mode types, rotational, translational, planet, and purely ring modes. Parker and $\mathrm{Wu}$ [18] mathematically proved modal properties of planetary gears having diametrically opposed planets and an elastic ring gear. Two types of modes were found, that is, rotational and translational modes. Canchi and Parker $[19,20]$ studied the parametric instability of rotating rings connected to moving discrete springs with time-varying stiffness. They analytically studied the effects of different system parameters on the instability boundaries and well-defined properties for the occurrence or suppression of instabilities are obtained as simple relations in the system parameters. Cooley and Parker [21] mathematically proved the structured properties of the critical speeds and associated critical speed eigenvectors of high-speed planetary gears. The results show that planetary gears have the only planet, rotational, and translational mode critical speeds. Divergence instability is possible at speeds adjacent to critical speeds, and flutter instabilities occur at extremely high speeds. Then, they [22] studied the vibration of high-speed rotating rings coupled to space-fixed stiffnesses. Zhao et al. [23] examined the parametric vibration of an elastic dual-ring structure by an analytical model including stationary supports and rotating loads. The results imply that there exist four types of excitations and that vibration can occur in a coupled, uncoupled, principal, or combination manner. Lu et al. [24] investigated the in-plane free vibration of high-speed rotating thin ring with an elastic foundation. Cooley and Parker [25] investigated eigenvalue sensitivity to model parameters and veering in gyroscopic systems by a perturbation approach. The results are shown to be accurate over a wide range of rotation speeds. A veering parameter is defined to analyze veering in high-speed planetary gears. Liu et al. [26] investigated the vibration of rotating elastic rings that are excited by an arbitrary number of space-fixed discrete stiffnesses with periodically fluctuating stiffnesses. The results show that the bandwidths of the instability regions correlate with the fractional strain energy stored in the discrete stiffnesses. For rings with multiple discrete stiffnesses, the phase differences between them can eliminate large amplitude response under certain conditions. Lu et al. [27] developed a new high-order model for in-plane vibrations of rotating rings. The study shows that the new high-order model is superior to the existing ring models in predicting the dynamic behavior of either stationary or rotating rings, and the model is applicable to both plane strain and plane stress configurations. In this paper, the through-thickness variation of the radial stress and geometrical nonlinearity were considered. As mentioned above, there has been no study on the vibration of planet rings, which have both rotating and revolving movements, with discrete stiffnesses and partially distributed stiffnesses. 
In this study, the vibration properties of high-speed rotating and revolving planet rings with discrete and partially distributed stiffnesses were investigated. The governing equations of motion for the radial and tangential deformations of planet rings were derived based on a rotating-basis system. This model considered the support zone variation and revolving movement of planet rings. Matrix differential operators were used to express the governing equations. The vibration properties of planet rings with and without stiffnesses were investigated by using the Galerkin method. The influence of parameters on vibration characteristics was studied. The forced responses excited by rotating and revolving movement were also investigated.

\section{Analytical Model}

The schematic of a planetary gear system and the vibration model of a planet ring are shown in Figure 1a. The rotating speed and revolving speed of the planet ring are $\omega$ and $\Omega$, respectively, and the ratio of $\Omega$ and $\omega$ is $i_{0}=\Omega / \omega$. The stationary basis is $\{\mathbf{i}, \mathbf{j}, \mathbf{k}\}$; the origin $O_{1}$ of $\left\{\mathbf{e}_{1}, \mathbf{e}_{2}, \mathbf{e}_{3}\right\}$ basis is set to the center of the ring, where the direction of $\mathbf{e}_{1}$ is along the extension of $O_{0} O_{1}$. The basis $\left\{\mathbf{e}_{1}{ }^{\prime}, \mathbf{e}_{2}{ }^{\prime}, \mathbf{e}_{3}{ }^{\prime}\right\}$ is a local coordinate system with the origin $\mathrm{O}_{2}$ on the middle surface, and $\mathbf{e}_{1}{ }^{\prime}$ is along the radial direction of the ring. $u(\theta \theta, t)$ and $v(\theta, t)$ are radial and tangential deformations, respectively. $M$ is a material point on the ring and $\phi$ is the relative angle of $\mathbf{e}_{1}{ }^{\prime}$ to the basis $\mathbf{e}_{1}$, where $\phi=\omega t+\theta, \theta$ is the specified angular coordinate of the point $M$ around the planet $\phi$ ring. $R_{0}$ is the revolving radii, and $R_{1}$ is the radii of the middle surface. The density of the ring is $p$, its elastic modulus is $E$, and its section area is $A$ with width $B$ and thickness $h$. Moreover, $k_{m 1}$ and $k_{m 2}$ are mesh stiffnesses between planet ring with sun gear and ring gear, respectively; $\alpha$ is the pressure angle of $k_{m i}$ on the ring, and apparently the mesh stiffnesses will affect both the radial and tangential deformations. When a planetary gear system runs, the contact zone between planet ring and rolling bearing usually concentrates on a part of the whole circle contact area [28] because of mesh forces. Hence, the planet ring is supported in the radial direction by discrete partially distributed stiffnesses, as shown in Figure $1 b$, and each spring denotes the position of a roller in the bearing and its position to $\mathbf{e}_{1}$ is $\theta_{b i}$. The support stiffness of the roller is $k_{b i}$, and the values of all $k_{b i}$ are assumed to be the same. The positions of $k_{m 1}$ and $k_{m 2}$ to $\mathbf{e}_{1}$ are $\theta_{m i}(i=1,2)$. Because the planet ring rotates, $\theta_{b i}$ and $\theta_{m i}$ are time dependent, and $\theta_{b i}=\theta_{b i 0}-\omega t, \theta_{m i}=\theta_{m i 0}-\omega t$, respectively, where $\theta_{b i 0}$ and $\theta_{m i 0}$ are the original angles to $\mathbf{e}_{1} \cdot \theta_{m i 0}$ is determined by the geometry of mesh pairs in the planetary gear system, and $\theta_{b i 0}$ is determined by the selected bearing.

The velocity vector of the point $M$ on the ring is obtained by the following kinetic formula [29]:

$$
\begin{aligned}
\mathbf{v}_{M} & =\mathbf{v}_{O_{1}}+\Omega \mathbf{e}_{3} \times \mathbf{r}_{M}+\mathbf{v}_{M, r} \\
& =R_{0} \Omega \mathbf{e}_{2}+\Omega \mathbf{e}_{3} \times\left(R_{1} \cos \phi \mathbf{e}_{1}+R_{1} \sin \phi \mathbf{e}_{2}+u \mathbf{e}_{1}^{\prime}+v \mathbf{e}_{2}^{\prime}\right)+\mathbf{v}_{M, r}
\end{aligned}
$$

where $\phi=\omega t+\theta, \mathbf{r}_{M}$ is the vector of $M$ in the coordinate $\left\{\mathbf{e}_{1}, \mathbf{e}_{2}, \mathbf{e}_{3}\right\}$, and $\mathbf{v}_{M, r}$ is the relative velocity of $M$ in the coordinate $\left\{\mathbf{e}_{1}, \mathbf{e}_{2}, \mathbf{e}_{3}\right\}$, which can be expressed as follows:

$$
\mathbf{v}_{M, r}=\mathbf{v}_{O_{2}}+\omega \mathbf{e}_{3}^{\prime} \times \mathbf{r}_{M 1}+\mathbf{v}_{r}=R_{1} \omega \mathbf{e}_{2}^{\prime}+\omega \mathbf{e}_{3}^{\prime} \times\left(u \mathbf{e}_{1}^{\prime}+v \mathbf{e}_{2}^{\prime}\right)+\left(\dot{u} \mathbf{e}_{1}^{\prime}+\dot{v} \mathbf{e}_{2}^{\prime}\right)
$$

Consequently, $\mathbf{v}_{M}$ becomes the following:

$$
\mathbf{v}_{M}=R_{0} \Omega \mathbf{e}_{2}+\Omega \mathbf{e}_{3} \times\left(R_{1} \cos \phi \mathbf{e}_{1}+R_{1} \sin \phi \mathbf{e}_{2}+u \mathbf{e}_{1}^{\prime}+v \mathbf{e}^{\prime}\right)+(\dot{u}-\omega v) \mathbf{e}_{1}^{\prime}+\left(R_{1} \omega+\omega u+\dot{v}\right) \mathbf{e}_{2}^{\prime}
$$

Since the velocity, $\mathbf{v}_{M}$, in Equation (3) is represented by unit vectors of two coordinates, it is better to be represented in one coordinate. Thus, according to the coordinate transformation, the velocity of $M$ can be represented in the coordinate $\left\{\mathbf{e}_{1}{ }^{\prime}, \mathbf{e}_{2}{ }^{\prime}, \mathbf{e}_{3}{ }^{\prime}\right\}$ as follows:

$$
\begin{aligned}
& \mathbf{v}_{M}=v_{r} \mathbf{e}_{1}^{\prime}+v_{t} \mathbf{e}_{2}^{\prime} \\
& =\left[\dot{u}-v(\Omega+\omega)+R_{0} \Omega \sin \phi\right] \mathbf{e}_{1}^{\prime}+\left[\dot{v}+u(\Omega+\omega)+R_{1}(\Omega+\omega)+R_{0} \Omega \cos \phi\right] \mathbf{e}_{2}^{\prime}
\end{aligned}
$$


where $v_{r}$ is the radial velocity and $v_{t}$ is the tangential velocity. Then the kinetic energy of the ring is as follows:

$$
K_{E}=\frac{1}{2} \rho A R_{1} \int_{0}^{2 \pi}\left(v_{r}^{2}+v_{t}^{2}\right) d \theta
$$

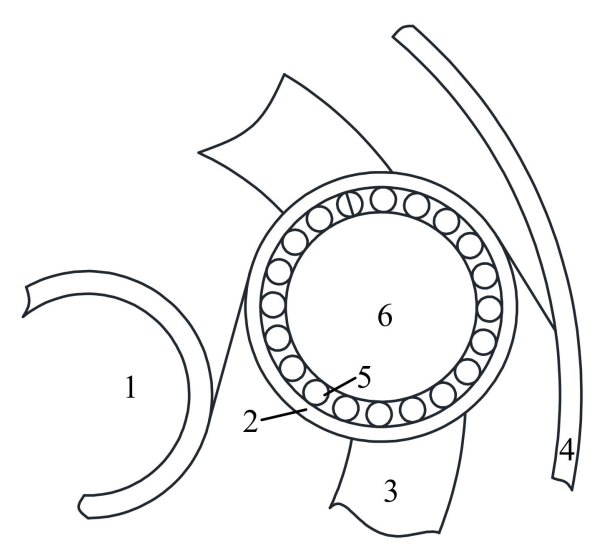

(a)

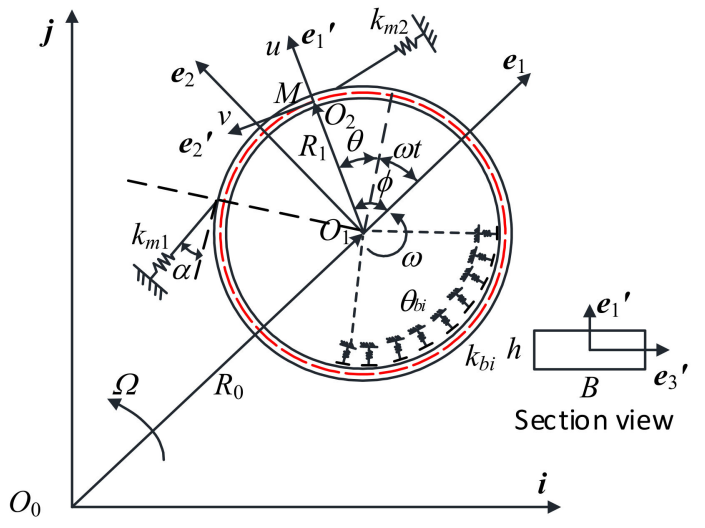

(b)

Figure 1. Schematic of planetary gear set: (a) physical model, 1-sun gear, 2-planet gear, 3-carrier, 4-ring gear, 5-bearing, 6-planet gear shaft; (b) vibration model of a planet ring.

Because the strain energy of the ring is independent of the rotating and revolving movement, the deformation of point $M$ can be considered in the coordinates $\left\{\mathbf{e}_{1}{ }^{\prime}, \mathbf{e}_{2}{ }^{\prime}, \mathbf{e}_{3}{ }^{\prime}\right\}$. The original length of a small segment of the middle surface of the ring is given as $\mathrm{d} S=$ $R_{1} \mathrm{~d} \theta$. After deformation, the vector $O_{1} M$, which is denoted by $\mathbf{r}$ and $\mathbf{r}=\left(R_{1}+u\right) \mathbf{e}_{1}{ }^{\prime}+v \mathbf{e}_{2}{ }^{\prime}$, will increase by $d r$. Then the length of the segment becomes the following:

$$
\mathrm{d} S^{\prime}=\sqrt{\mathrm{d} \mathbf{r} \cdot \mathrm{d} \mathbf{r}}=\sqrt{\left[\left(R_{1}+u+\partial v / \partial \theta\right) \mathrm{d} \theta\right]^{2}+[(\partial u / \partial \theta-v) \mathrm{d} \theta]^{2}}
$$

Therefore, the strain of the segment on the middle surface is as follows:

$$
\varepsilon_{0}=\frac{\mathrm{d} S^{\prime}-\mathrm{d} S}{\mathrm{~d} S}=\frac{\sqrt{\left[\left(R_{1}+u+\partial v / \partial \theta\right) \mathrm{d} \theta\right]^{2}+[(\partial u / \partial \theta-v) \mathrm{d} \theta]^{2}}-R_{1} \mathrm{~d} \theta}{R_{1} \mathrm{~d} \theta}
$$

Here, Equation (7) is approximated to second-order, and it gives the following:

$$
\varepsilon_{0}=\frac{1}{R_{1}}\left(u+\frac{\partial v}{\partial \theta}\right)+\frac{1}{2 R_{1}^{2}}\left(v-\frac{\partial u}{\partial \theta}\right)^{2}
$$

The strain of any material point on the cross-section of the ring can be obtained by linear assumption:

$$
\varepsilon=\varepsilon_{0}+\frac{r^{\prime}}{R_{1}^{2}}\left(\frac{\partial v}{\partial \theta}-\frac{\partial^{2} u}{\partial \theta^{2}}\right)
$$

where $r^{\prime}$ is the radial distance of a point on the cross-section to the middle surface. Therefore, the total strain energy of the ring is as follows:

$$
S_{e}=\frac{1}{2} \iint_{A \theta} \sigma \varepsilon d A R_{1} \mathrm{~d} \theta
$$

where $\sigma$ is the stress. The actions of partially distributed and discrete stiffnesses are considered as external forces to the ring; therefore, the work of the stiffnesses is as follows: 


$$
W_{s}=\int_{\theta}\left[\left(F_{b}+F_{m r}\right) u+F_{m t} v\right] d \theta
$$

where $F_{b}$ is elastic forces of partially distributed stiffnesses, and $F_{m r}$ and $F_{m t}$ are radial and tangential components of discrete stiffnesses acting on the ring, respectively.

Accordingly, the governing equations can be derived by Hamilton's principle:

$$
\delta \int_{t_{0}}^{t_{1}}\left(K_{E}-S_{e}+W_{s}\right) \mathrm{d} t=0
$$

By substituting Equations (5), (10), and (11) into Equation (12) and ignoring the nonlinear terms for the small vibrations, we get the governing equations of vibration of the planet ring:

$$
\begin{aligned}
& \rho A R_{1} \frac{\partial^{2} u}{\partial t^{2}}-2 \rho A R_{1}(\Omega+\omega) \frac{\partial v}{\partial t}-\rho A R_{1}(\Omega+\omega)^{2} u+N-\frac{\partial^{2} M}{R_{1} \partial \theta^{2}}+F_{b}+F_{m r} \\
& =\rho A R_{1}^{2}(\Omega+\omega)^{2}+\rho A R_{1} R_{0} \Omega^{2} \cos \phi \\
& \quad \rho A R_{1} \frac{\partial^{2} v}{\partial t^{2}}+2 \rho A R_{1}(\Omega+\omega) \frac{\partial u}{\partial t}-\rho A R_{1}(\Omega+\omega)^{2} v-\frac{\partial N}{\partial \theta}-\frac{\partial M}{R_{1} \partial \theta}+F_{m t} \\
& \quad=-\rho A R_{1} R_{0} \Omega^{2} \sin \phi
\end{aligned}
$$

where $N$ and $M$ are the vibration-induced force and moment resultants, respectively:

$$
\begin{gathered}
N=E A\left(\frac{u}{R_{1}}+\frac{\partial v}{R_{1} \partial \theta}\right) \\
M=\frac{E I}{R_{1}^{2}}\left(\frac{\partial v}{\partial \theta}-\frac{\partial^{2} u}{\partial \theta^{2}}\right)
\end{gathered}
$$

and

$$
\begin{gathered}
F_{b}=\sum_{i=1}^{N_{b}}\left[F_{b i} \Delta\left(\theta-\theta_{b i}\right)\right]=\sum_{i=1}^{N_{b}}\left[k_{b i} u\left(\theta_{b i}, t\right)\right] \Delta\left(\theta-\theta_{b i}\right) \\
F_{m r}=\sum_{i=1}^{N_{m}}\left[F_{m r i} \Delta\left(\theta-\theta_{m i}\right)\right]=\sum_{i=1}^{N_{m}}\left[k_{m i} u\left(\theta_{m i}, t\right) \sin \alpha\right] \Delta\left(\theta-\theta_{m i}\right) \\
F_{m t}=\sum_{i=1}^{N_{m}}\left[F_{m t i} \Delta\left(\theta-\theta_{m i}\right)\right]=\sum_{i=1}^{N_{m}}\left[k_{m i} v\left(\theta_{m i}, t\right) \cos \alpha\right] \Delta\left(\theta-\theta_{m i}\right)
\end{gathered}
$$

$F_{b i}, F_{m r i}$, and $F_{m t i}$ are forces of the $i$ th partially distributed stiffness, radial, and tangential components of discrete stiffness, respectively. $N_{b}$ and $N_{m}$ are the numbers of partially distributed stiffnesses and discrete stiffnesses, respectively. $\theta_{b i}, \theta_{m i}$ are the corresponding time dependent positions of partially distributed stiffnesses and discrete stiffnesses, respectively. $\Delta(\cdot)$ is the Dirac delta function. It can be seen from the right side of Equation (13) that the rotation and revolving movements will result in constant centrifugal force, $\rho A R_{1}^{2}(\Omega+\omega)^{2}$, and time-dependent excitation, $\rho A R_{1} R_{0} \Omega^{2} \cos \phi, \rho A R_{1} R_{0} \Omega^{2} \sin \phi$, to the ring.

For the steady configuration, the ring has no tangential deflection because there is no steady force in this direction, as shown in Equation (13b). Therefore, the radial steady equations of the ring can be obtained from Equation (13a) by the elimination of the time derivatives:

$$
\begin{gathered}
\frac{E I}{R_{1}^{3}} \frac{\partial^{4} u_{e}}{\partial \theta^{4}}+\left(\frac{E A}{R_{1}}-\rho A R_{1} i_{0}^{\prime} \omega^{2}\right) u_{e}+\sum_{i=1}^{N_{b}}\left[k_{b i} u_{e}\left(\theta_{b i}\right)\right] \Delta\left(\theta-\theta_{b i}\right) \\
+\sum_{i=1}^{N_{m}}\left[k_{m i} u_{e}\left(\theta_{m i}\right) \sin \alpha\right] \Delta\left(\theta-\theta_{m i}\right)=\rho A R_{1}^{2} i_{0}^{\prime} \omega^{2}
\end{gathered}
$$


where $u_{e}$ denotes the steady radial deformation and $i_{0}^{\prime}=\left(i_{0}+1\right)^{2}$. Moreover, $u_{e}$ in the nonlinear Equation (16) can be solved by numerical method.

To estimate the critical speed analytically, the high-order differentials and stiffnesses of Equation (16) are ignored, and the estimated steady radial deformation can be obtained from Equation (16) as follows:

$$
u_{e}=\frac{\rho A R_{1}^{2} i_{0}^{\prime} \omega^{2}}{\frac{E A}{R_{1}}-\rho A R_{1} i_{0}^{\prime} \omega^{2}}
$$

It can be found that the steady ring radial deformation in Equation (17) becomes extremely large when the speed, $\omega$, is close to $\omega_{c}$ :

$$
\omega_{c}=\sqrt{E A / \rho A R_{1}^{2} i_{0}^{\prime}}
$$

When the speed, $\omega$, is larger than $\omega_{c}$, the steady status in Equation (17) is unstable. By applying the parameters in Table 1 in Section 3, the critical speed, $\omega_{c}$, calculated by Equation (16) and Equation (18), is $5.4339 \times 10^{4}$ and $5.4324 \times 10^{4}$, respectively. Therefore, it is reasonable to roughly estimate the critical speed of Equation (16) by the simplified Equation (18). In this work, the speed is restricted lower than that of Equation (18).

Table 1. Parameters of a planet ring with discrete and partially distributed stiffnesses.

$\begin{array}{cc}\text { Ring radius } R_{0}(\mathrm{~mm}) & 100 \\ \text { Ring radius } R_{1}(\mathrm{~mm}) & 80 \\ \text { Cross section area } A\left(\mathrm{~mm}^{2}\right) & 250 \\ \text { Area moment of inertia } I\left(\mathrm{~mm}^{4}\right) & 2083 \\ \text { Pressure Angle } \alpha\left({ }^{\circ}\right) & 20 \\ \text { Elastic modulus } E(\mathrm{GPa}) & 206 \\ \text { Density } p\left(\mathrm{~kg} / \mathrm{m}^{3}\right) & 7800 \\ \text { Mean mesh stiffness } k_{m i}(\mathrm{~N} / \mathrm{m}) & 5 \times 10^{8} \\ \text { Support stiffness } k_{b i}(\mathrm{~N} / \mathrm{m}) & 1 \times 10^{8} \\ \text { The ratio } i_{0} \text { of } \Omega \text { to } \omega & 0.1825\end{array}$

Equation (13) can be rewritten to be the equations of small vibrations about the steady status by substitution of $u \rightarrow u_{e}+u$. After that, Equation (13) is normalized by the following variables, to investigate the general vibration properties of planet rings:

$$
\omega_{0}=\frac{1}{R_{1}^{2}} \sqrt{\frac{E I}{\rho A}}, \bar{u}, \bar{v}=\frac{u, v}{R_{1}}, \bar{t}=\omega_{0} t, \bar{\omega}, \bar{\Omega}=\frac{\omega, \Omega}{\omega_{0}}, \bar{k}_{b i}, \bar{k}_{m i}=\frac{k_{b i}, k_{m i} R_{1}^{3}}{E I}, \gamma=\frac{E A R_{1}^{2}}{E I}
$$

The dimensionless equations are obtained by substitution of Equation(19):

$$
\begin{gathered}
\frac{\partial^{2} u}{\partial t^{2}}-2(\Omega+\omega) \frac{\partial v}{\partial t}-(\Omega+\omega)^{2} u+\gamma\left(u+\frac{\partial v}{\partial \theta}\right)+\left(\frac{\partial^{4} u}{\partial \theta^{4}}-\frac{\partial^{3} v}{\partial \theta^{3}}\right) \\
+\sum_{i=1}^{N_{b}}\left[k_{b i} u\left(\theta_{b i}, t\right)\right] \Delta\left(\theta-\theta_{b i}\right)+\sum_{i=1}^{N_{m}}\left[k_{m i} u\left(\theta_{m i}, t\right) \sin \alpha\right] \Delta\left(\theta-\theta_{m i}\right)=\frac{R_{0}}{R_{1}} \Omega^{2} \cos \phi \\
\frac{\partial^{2} v}{\partial t^{2}}+2(\Omega+\omega) \frac{\partial u}{\partial t}-(\Omega+\omega)^{2} v-\gamma\left(\frac{\partial u}{\partial \theta}+\frac{\partial^{2} v}{\partial \theta^{2}}\right)+\left(\frac{\partial^{3} u}{\partial \theta^{3}}-\frac{\partial^{2} v}{\partial \theta^{2}}\right) \\
+\sum_{i=1}^{N_{m}}\left[k_{m i} v\left(\theta_{m i}, t\right) \cos \alpha\right] \Delta\left(\theta-\theta_{m i}\right)=-\frac{R_{0}}{R_{1}} \Omega^{2} \sin \phi
\end{gathered}
$$

The right parts of Equation (20) are external excitations resulting from rotating and revolving movement. In Equation (20), the bar on variables is removed for convenience, and Equation (20) is rewritten in matrix form by partial differential operators, as follows:

$$
\mathbf{M} \ddot{\mathbf{x}}+(\Omega+\omega) \mathbf{G} \dot{\mathbf{x}}+\mathbf{K} \mathbf{x}=\mathbf{F}
$$


where

$$
\begin{aligned}
& \mathbf{x}=\left\{\begin{array}{ll}
u & v
\end{array}\right\}^{\mathrm{T}}, \mathbf{M}=\left[\begin{array}{ll}
1 & 0 \\
0 & 1
\end{array}\right], \mathbf{G}=\left[\begin{array}{cc}
0 & -2 \\
2 & 0
\end{array}\right], \mathbf{F}=\left\{\frac{R_{0}}{R_{1}} \Omega^{2} \cos \phi,-\frac{R_{0}}{R_{1}} \Omega^{2} \sin \phi\right\}^{\mathrm{T}},
\end{aligned}
$$

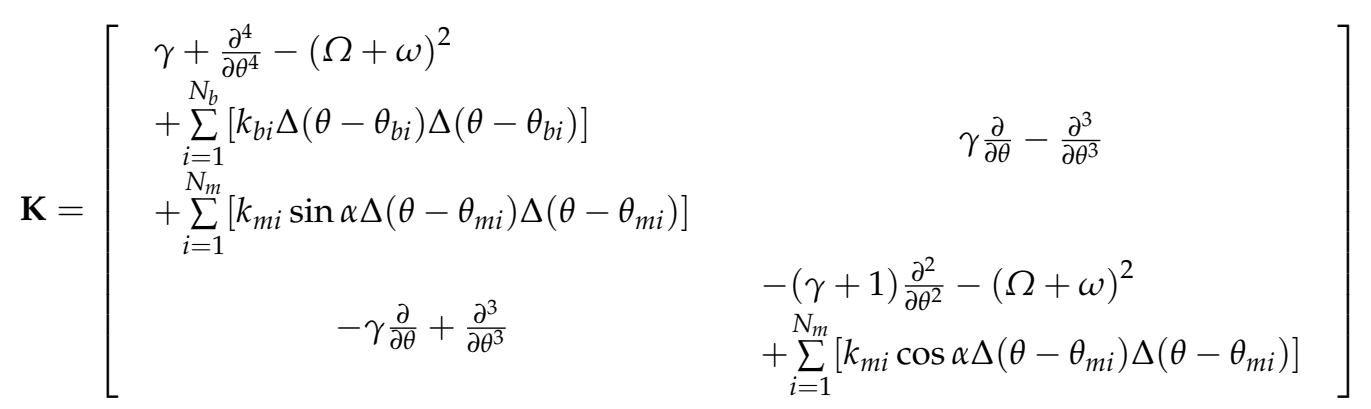

In Equation (21), the components of $\mathbf{F}$ are functions of $\theta$ and $t$. Obviously, they are the excitation forces in the radial and tangential directions, which are caused by rotating and revolving movement. At the left side of Equation (21), the rotating and revolving velocities are all involved. Therefore, the revolving movement will affect the free vibration of the ring.

\subsection{Natural Characteristics}

To obtain natural frequencies and modes of the planet ring, the external excitation, $F$, is set to 0 . By using a separable solution $\mathbf{x}=\chi \mathrm{e}^{\lambda t}$, the corresponding eigenvalue problem becomes the following:

$$
\lambda^{2} \mathbf{M} \boldsymbol{\chi}+\lambda(\Omega+\omega) \mathbf{G} \boldsymbol{\chi}+\mathbf{K} \boldsymbol{\chi}=0
$$

For the non-axisymmetric discrete and partially distributed stiffnesses on the planet ring, the eigenvectors are not single nodal diameter (ND) modes anymore. Therefore, Galerkin's method is applied to solve the natural frequencies and their modes for this nonaxisymmetric case. The eigenvectors are given as a series of linearly independent vectors:

$$
\boldsymbol{\chi}=\left\{\sum_{n=-N}^{N} c_{n} \phi_{n} \sum_{m=-M}^{M} d_{m} \varphi_{m}\right\}^{\mathrm{T}}
$$

where $2 N+1$ and $2 M+1$ are the numbers of orders of radial basis function, $\phi_{n}$, and tangential base function, $\varphi \varphi_{m}$, in Equation (23), respectively. The weight function $\chi^{\prime}=$ $\left[\begin{array}{cccc}\bar{\phi}_{j} & 0 ; & 0 & \bar{\varphi}_{k} ;\end{array}\right]^{\mathrm{T}}$ is used to construct the Galerkin target function, and the equations are expressed in matrix form, with a size of $2(N+M+1) \times 2(N+M+1)$ :

$$
\lambda^{2}[\mathbf{M}] \mathbf{x}+\lambda(\Omega+\omega)[\mathbf{G}] \mathbf{x}+[\mathbf{K}] \mathbf{x}=0
$$

where $\mathbf{x}=\left\{c_{-N}, \ldots, c_{N}, d_{-M}, \ldots, d_{M}\right\}^{\mathrm{T}}$,

$$
\begin{gathered}
{[\mathbf{M}]=\left[\begin{array}{cc}
{\left[\mathbf{M}_{u}\right]} & 0 \\
0 & {\left[\mathbf{M}_{v}\right]}
\end{array}\right],[\mathbf{G}]=\left[\begin{array}{cc}
0 & -\left[\mathbf{G}_{u v}\right] \\
{\left[\mathbf{G}_{v u}\right]} & 0
\end{array}\right],[\mathbf{K}]=\left[\begin{array}{cc}
{\left[\mathbf{K}_{u}+\mathbf{K}_{b}+\mathbf{K}_{m r}\right]} & {\left[\mathbf{K}_{u v}\right]} \\
{\left[\mathbf{K}_{v u}\right]} & {\left[\mathbf{K}_{v}+\mathbf{K}_{m t}\right]}
\end{array}\right],} \\
\mathbf{M}_{u}(j, n)=\int_{0}^{2 \pi} \bar{\phi}_{j} \phi_{n} \mathrm{~d} \theta, \mathbf{M}_{v}(k, m)=\int_{0}^{2 \pi} \bar{\varphi}_{k} \varphi_{m} \mathrm{~d} \theta \\
\mathbf{G}_{u v}(j, m)=\int_{0}^{2 \pi} 2 \bar{\phi}_{j} \varphi_{m} \mathrm{~d} \theta, \mathbf{G}_{v u}(k, n)=\int_{0}^{2 \pi} 2 \bar{\varphi}_{k} \phi_{n} \mathrm{~d} \theta \\
\mathbf{K}_{u}(j, n)=\int_{0}^{2 \pi}\left(\left[\gamma-(\Omega+\omega)^{2}\right] \bar{\phi}_{j} \phi_{n}+\bar{\phi}_{j} \phi_{n}^{\prime \prime \prime \prime}-N_{c}^{\prime} \bar{\phi}_{j} \phi_{n}^{\prime \prime}\right) \mathrm{d} \theta \\
\mathbf{K}_{u v}(j, m)=\int_{0}^{2 \pi}\left(\gamma \bar{\phi}_{j} \varphi_{m}^{\prime}-\bar{\phi}_{j} \varphi_{m}^{\prime \prime \prime}\right) \mathrm{d} \theta, \mathbf{K}_{v u}(k, n)=\int_{0}^{2 \pi}\left(-\gamma \bar{\varphi}_{k} \phi_{n}^{\prime}+\bar{\varphi}_{k} \phi_{n}^{\prime \prime \prime}\right) \mathrm{d} \theta \\
\mathbf{K}_{v}(k, m)=\int_{0}^{2 \pi}\left[-(\gamma+1) \bar{\varphi}_{k} \varphi_{m}^{\prime \prime}-(\Omega+\omega)^{2} \bar{\varphi}_{k} \varphi_{m}\right] \mathrm{d} \theta
\end{gathered}
$$




$$
\begin{gathered}
\mathbf{K}_{b}(j, n)=\int_{0}^{2 \pi} \sum_{i=1}^{N_{b}} k_{b i} \bar{\phi}_{j}\left(\theta_{b i}, t\right) \phi_{n}\left(\theta_{b i}, t\right) \mathrm{d} \theta, \\
\mathbf{K}_{m r}(j, n)=\int_{0}^{2 \pi} \sum_{i=1}^{N_{b}} k_{m i} \sin \alpha \bar{\phi}_{j}\left(\theta_{m i}, t\right) \phi_{n}\left(\theta_{m i}, t\right) \mathrm{d} \theta, \\
\mathbf{K}_{m t}(k, m)=\int_{0}^{2 \pi} \sum_{i=1}^{N_{m}} k_{m i} \cos \alpha \bar{\phi}_{k}\left(\theta_{m i}, t\right) \phi_{m}\left(\theta_{m i}, t\right) \mathrm{d} \theta
\end{gathered}
$$

After substituting Equation (23) into Equation (22), the order numbers of radial and circumferential basis functions are $2 N+1$ and $2 M+1$, respectively. According to the Galerkin method, each formula is multiplied by the conjugate function of each corresponding basis function and then integrated. Thus, $2(N+M+1) \times 2(N+M+1)$ formulas can be obtained, namely the size of matrices [M], [G], and [K] are $2(N+M+1) \times 2(N+M+1)$. Each element of these matrices can be calculated according to the element formula under Equation (24). Choosing proper basis functions $\phi_{n}(\theta)$ and $\varphi_{m}(\theta)$, the eigenvalue problem of Equation (24) can be solved following the state space eigenvalue problem, as described in Reference [30], and the numerical calculation can be made by standard methods.

\subsection{Forced Response}

Equation (21) reveals that the rotating and revolving movement will result in external excitation to the planet ring. Accordingly, the forced response of the planet ring to rotating and revolving movement were investigated. The forced response is therefore assumed to be of the following form:

$$
\begin{aligned}
& u(\theta, t)=\sum_{n=0}^{N_{f}-1}\left[p_{n}(t) \cos n \theta+q_{n}(t) \sin n \theta\right] \\
& v(\theta, t)=\sum_{n=0}^{N_{f}-1}\left[\xi_{n}(t) \cos n \theta+\eta_{n}(t) \sin n \theta\right]
\end{aligned}
$$

where $p_{n}(t), q_{n}(t), \xi_{n}(t), \eta_{n}(t)$ are the generalized coordinates, $N_{f}$ is the number of truncated modal order. Equation (25) is inserted into Equation (21), and the orthogonality of $\cos (n \theta)$ and $\sin (n \theta)$ in a period interval of $2 \pi$ is then used to discretize the equations. The equations are expressed in matrix form, as follows:

$$
\widetilde{\mathbf{M}} \ddot{\mathbf{y}}+\widetilde{\mathbf{G}} \dot{\mathbf{y}}+\widetilde{\mathbf{K}} \mathbf{y}=\widetilde{\mathbf{Q}}
$$

where $\mathbf{y}=\left\{\begin{array}{llll}p_{n}(t) & q_{n}(t) & \xi_{n}(t) & \eta_{n}(t)\end{array}\right\}^{\mathrm{T}} ; \widetilde{\mathbf{M}}, \widetilde{\mathbf{G}}, \widetilde{\mathbf{K}}$ are the matrices with order $4 N_{f} \times$ $4 N_{f}$; and the excitation vector, $\widetilde{\mathbf{Q}}$, is written in Appendix A. Accordingly, the forced response of Equation (21) can be obtained by Equation (26) with the solution of Equation (25).

\section{Vibration Analysis}

To analyze the natural vibration properties of high-speed rotating and revolving planet rings with discrete and partially distributed stiffnesses, basis functions $\phi_{n}=e^{j n \theta}, \varphi_{m}=e^{j m \theta}$ were chosen. Since this work is motivated by planetary gear systems, where the planet gear is an elastic thin ring acted by discrete gear meshes and support bearing, the parameters of the planet ring in a planetary gear system are given in Table 1 . Since there is no reference to study the vibration of planet rings, the model in Figure 1 is degenerated by ignoring the revolving movement to validate the equations and programming of our work with Reference [22]. By using the parameters in Reference [22], eigenvalue loci of a free and rotating elastic ring for varying rotation speeds are calculated by our degenerated model, and the result is shown in Figure 2. It can be found that the result is the same as the result of Figure 4 in Reference [22]. 


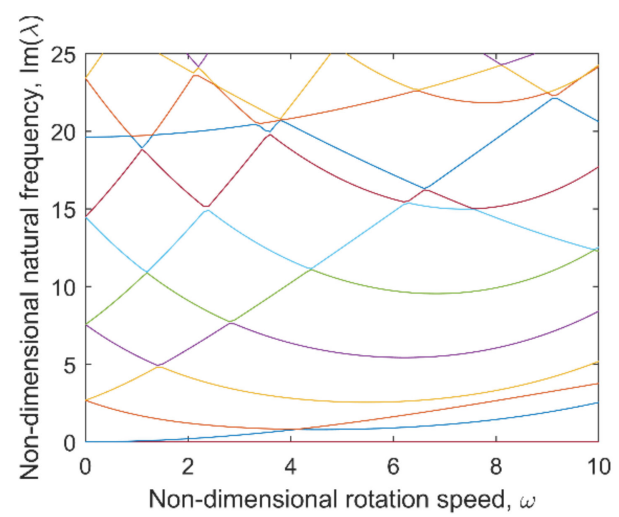

Figure 2. The dimensionless natural frequencies with respect to dimensionless rotation speed with the parameters in Reference [22].

\subsection{Free Planet Ring}

In practical applications, such as planetary gear systems, planet rings behave like free rings in idle status. Figure 3 a shows the natural frequencies of a free planet ring, with parameters in Table 1 calculated by the Galerkin method. The natural frequencies have degeneracy two, which is typical in axisymmetric spinning systems, like spinning disks [31] and spinning disk-spindle systems [32]. Two natural frequencies with the same nodal diameter separate at non-zero rotation speed and become different as shown in Figure 3a. One represents vibration waves propagating in the positive $\theta$ direction, and the other represents vibration waves propagating in the negative $\theta$ direction, which corresponds to backward waves and forward waves, respectively. Moreover, the corresponding vibration mode for all the frequencies is dominated by only one nodal diameter. The representative vibration mode and its components at $\omega=1$ are shown in Figure $3 \mathrm{~b}$. The eigenvalues for the system are purely imaginary over the range of analyzed speeds. It can be found that $1 \mathrm{ND}$ backward frequencies intersect with abscissa at point $C$ in Figure 3a. This indicates that possible unstable phenomena exist and the value at point $C$ is the critical speed.

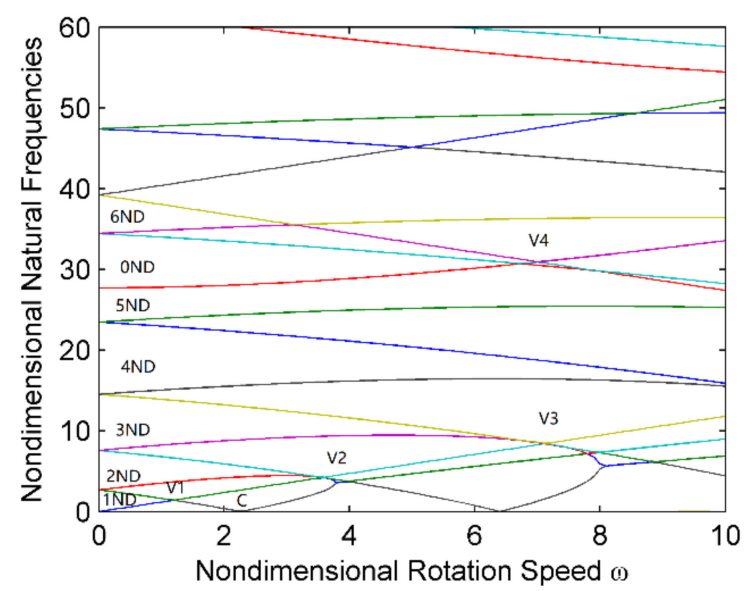

(a)
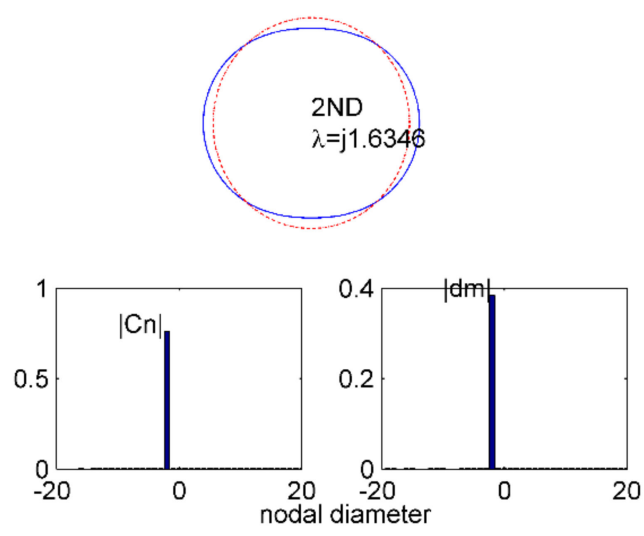

(b)

Figure 3. (a) Natural frequencies of a free planet ring versus varying speed; (b) representative vibration mode and its components at speed $\omega=1$ (dash line = original position; solid line = vibration mode).

Frequency veering occurs in multiple regions with the increase of rotation speed, such as the areas V1, V2, V3, and V4 in Figure 3a, and V2 for 1ND and 2ND at $\omega=3.5$, etc. In these regions, the lower ND frequencies become larger than the higher ND frequencies, that is, the modes exchange here. Figure 4 shows the modes exchange with the frequency veering at $\mathrm{V} 2$ around $\omega=3.5$. All the other veering regions have similar behavior. This 
means that, with the increase of rotation speed, the high-order frequencies may be dominated by lower ND components in the vibration mode, and, conversely, the low-order frequencies may be dominated by high ND components.

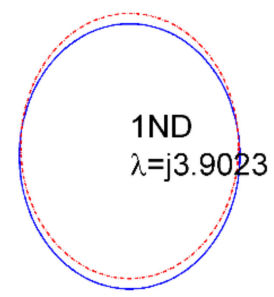

(a)

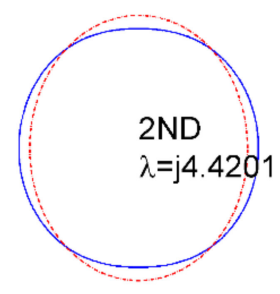

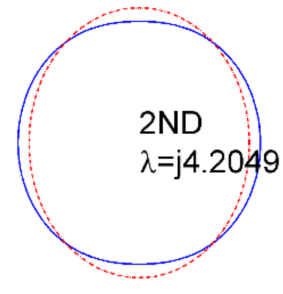

(b)

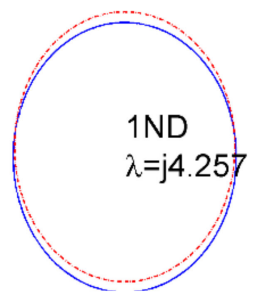

Figure 4. Frequency veering with vibration mode exchange between (a) $\omega=3.4$ and (b) $\omega=3.6$. The dash and solid lines denote the original position and vibration modes, respectively.

\subsection{Planet Ring with Stiffnesses}

Figure 5a shows the natural frequencies of a planet ring with discrete stiffnesses and partially distributed stiffnesses in the original region from $-\pi$ to 0 . There are also frequency veering regions as the free planet ring shows. Since the discrete and partially distributed stiffnesses disrupt the axisymmetry of the ring, the eigenvalues have distinct properties at $\omega=0$, compared to Figure 3a. For instance, in the regions where $\omega=0$, there are no the same natural frequencies of any two vibration modes, which is quite different from that of free planet rings. The number of frequency veering regions is less than free planet rings. In addition, the natural frequencies are much higher than free planet rings because the discrete and partially distributed stiffnesses enhance the stiffness of the whole ring. Figure $5 \mathrm{~b}$ illustrates the typical vibration modes (2nd to 6 th order modes) of planet rings with discrete and partially distributed stiffnesses. It is obvious that the partially distributed stiffnesses partially constrain the vibration of planet rings, as shown in Figure 5b. Meanwhile, the vibration modes are dominated by several components, as shown in Figure 5b, instead of a single component in free planet rings.

\subsection{Influences of Parameters}

The influences of several parameters, such as the number of partially distributed stiffnesses (NPDS), area of partially distributed stiffnesses, and discrete and partially distributed stiffnesses, on the vibration properties of planet rings are investigated in the following.

Different types of bearings with the same outer diameter may have different numbers of rollers or balls. Therefore, the influence of numbers of balls or rollers, that is, the number of partially distributed stiffnesses, is analyzed. Figure 6 shows the natural frequencies of a planet ring with discrete stiffnesses and changing the number of partially distributed stiffnesses. With the increase of the number of partially distributed stiffnesses, the natural frequencies increase, as it increases the stiffness of the whole ring partially. Moreover, from Figure 6, it can be observed that the lower frequencies are affected slightly, while the higher frequencies are increasing sharply with NPDS. Therefore, it could be a method for designers to avoid some natural frequencies by changing the number of partially distributed stiffnesses. 


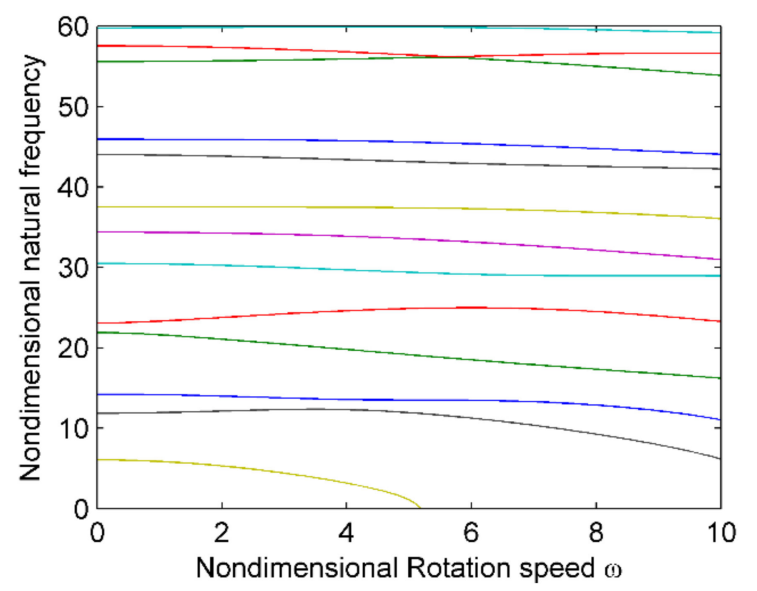

(a)
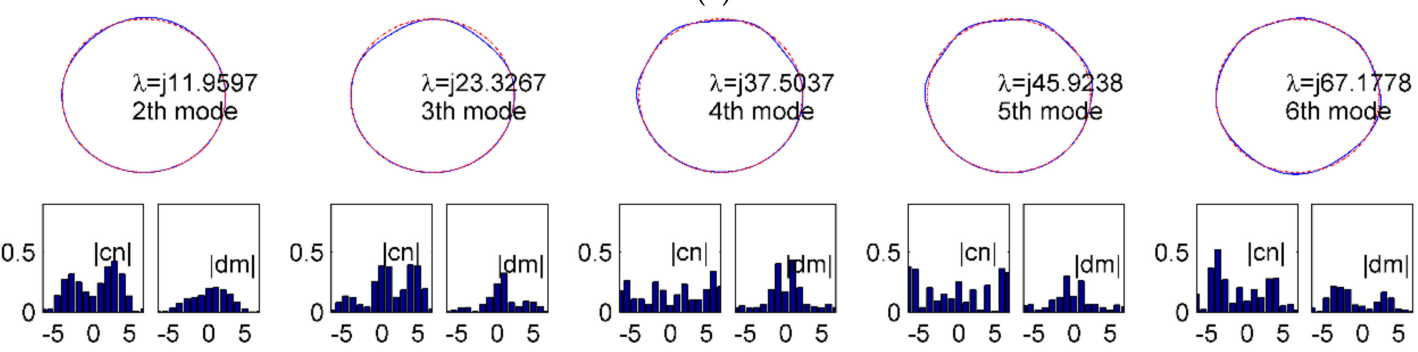

(b)

Figure 5. Natural frequencies and vibration modes of a planet ring with discrete and partial distributed stiffnesses $\left(N_{f}=20\right)$ : (a) natural frequencies vs. rotation speed $\omega$; (b) typical vibration modes of planet rings at $\omega=1$ (dash line $=$ original position; solid line = vibration mode).

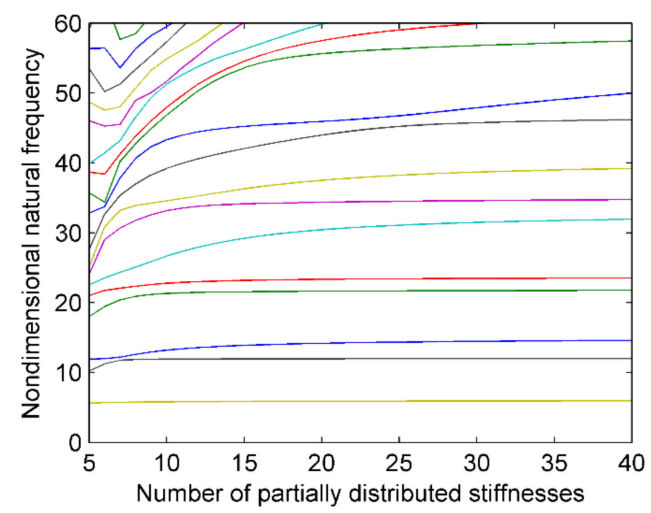

Figure 6. Natural frequencies of a planet ring with the different number of partially distributed stiffnesses at $\omega=1$.

In applications of planetary gear systems, the loads on the gears may change at different working status. The load changing on the planet gear rings may change the contact area with the bearings, that is, the number of partially distributed stiffnesses. To analyze the influence, the number of rolling elements is set to 36 , and the change range of the area is set as $90^{\circ} \sim 180^{\circ}$. Figure 7 shows the natural frequency variation versus partially distributed area at a rotating speed of $\omega=1$. Figure 7 reveals that the support area will influence the natural frequencies heavily. The natural frequencies are all increase with the increase of support area, and it especially has a great influence on the higher-order natural frequencies. This means, in heavy load conditions, the natural frequencies will get lower, and in light load conditions, the natural frequencies will get higher. 


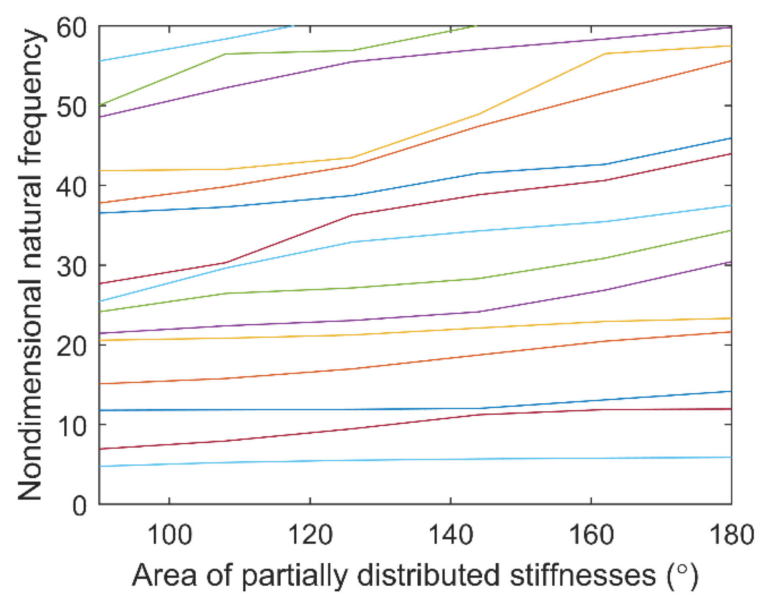

Figure 7. Dimensionless natural frequencies of a planet ring vs. area of partially distributed stiffnesses at $\omega=1$.

Figure 8a shows the influence of partially distributed stiffnesses to natural frequencies. It can be seen that only higher-order frequencies are affected largely by partially distributed stiffnesses. Moreover, in the analysis range of partially distributed stiffnesses, there is no obvious frequency veering phenomenon in Figure 8a. To analyze the mode veering properties, a veering parameter, $\xi_{m n}$, is chosen, as done in Reference [25], and can be calculated as follows. Equation (24) can be rewritten in the state space form, as follows:

$$
\lambda \mathbf{A x}+\mathbf{B} \mathbf{x}=0
$$

where

$$
\boldsymbol{x}=[\lambda \mathbf{x}, \mathbf{x}]^{\mathrm{T}}, \mathbf{A}=\left[\begin{array}{cc}
\mathbf{M} & 0 \\
0 & \mathbf{K}
\end{array}\right], \mathbf{B}=\left[\begin{array}{cc}
(\Omega+\omega) \mathbf{G} & \mathbf{K} \\
-\mathbf{K} & 0
\end{array}\right]
$$

then

$$
\xi_{m n}=\frac{\left\langle\chi_{m}, \lambda_{m} \mathbf{A}_{1} \chi_{n}+\mathbf{B}_{1} \chi_{n}\right\rangle\left\langle\chi_{n}, \lambda_{n} \mathbf{A}_{1} \chi_{n}+\mathbf{B}_{1} \boldsymbol{\chi}_{m}\right\rangle}{\lambda_{m}-\lambda_{n}}
$$

where $m, n$ denotes the order of frequencies; $<\bullet, \bullet>$ denotes the inner production; $\mathbf{A}_{1}$ and $\mathbf{B}_{1}$ are the first derivative of $A$ and $B$, with respect parameters such as stiffness here. For clarity, only 5-10 order veering parameters are calculated as shown in Figure 8c. It can be found that mode veering can be indicated by the veering parameter. Moreover, mode veering mainly appears in the range of smaller partial distributed stiffnesses. Figure $8 \mathrm{~b}$ shows the influence of discrete stiffnesses on natural frequencies. As seen in Figure $8 \mathrm{~b}$ a majority of frequencies are affected slightly by the changing of discrete stiffnesses and only a few frequencies are affected comparatively large. Besides, since the discrete stiffnesses are time-varying in gear systems, the influence of time-varying of discrete stiffnesses can also be analyzed by Figure $8 \mathrm{~b}$. Since the time-varying part of discrete stiffnesses is very small compared to their mean value, its influence is only a small segment of abscissa in Figure $8 \mathrm{~b}$. It also can be found that there is no mode veering in a large range of discrete stiffnesses, and mode veering appears only when discrete stiffness is very small. 


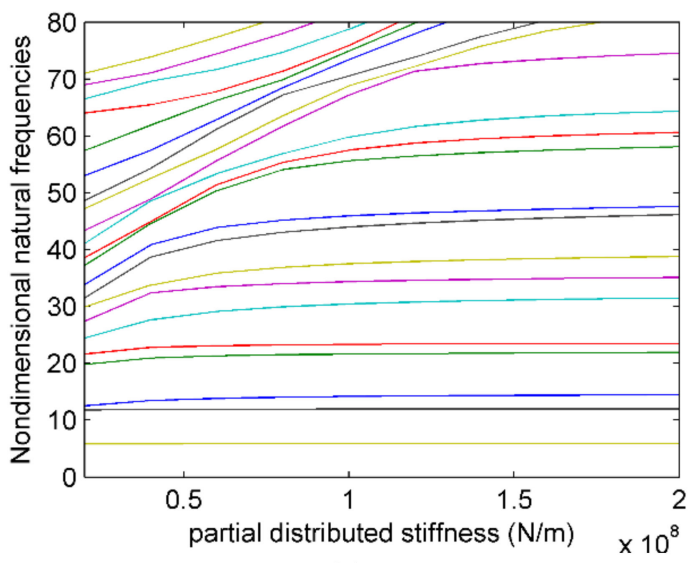

(a)

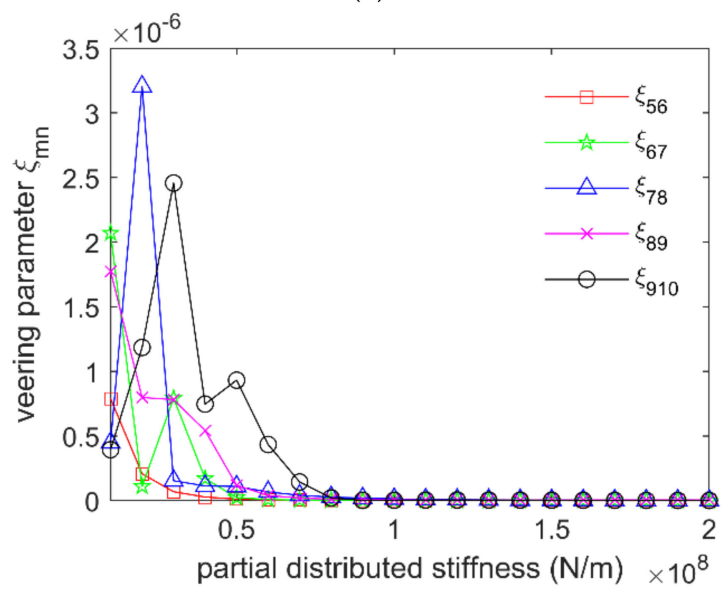

(c)

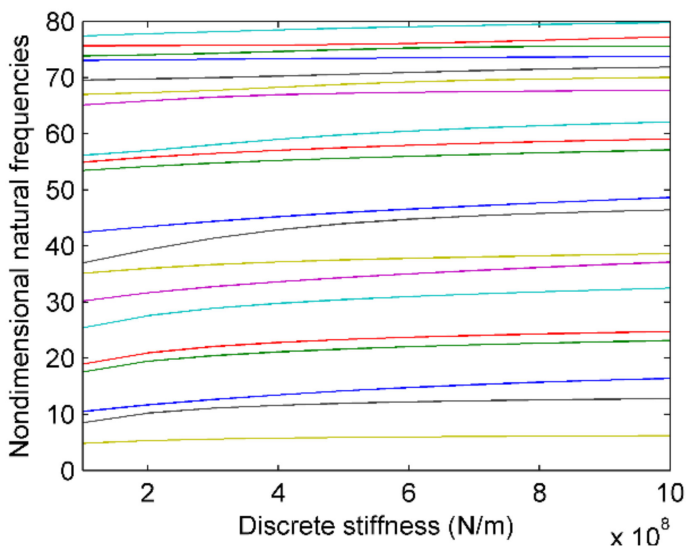

(b)

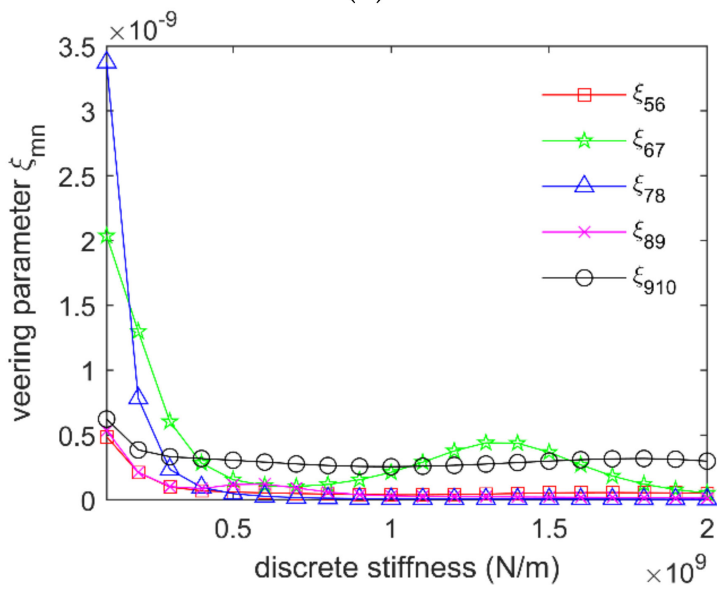

(d)

Figure 8. Influences of (a) partial distributed stiffness, (b) discrete stiffness to natural frequencies, (c) veering parameter $\xi_{m n}$ for (a), and (d) veering parameter $\xi_{m n}$ for $(\mathbf{b})$ at $\omega=1$.

\subsection{Forced Response}

In a planetary gear set, the rotating speed and revolving speed of a planet ring are proportional. In this work, the ratio is set to $i_{0}=0.1825$, according to a practical application. Therefore, the excitation of rotating and revolving movement to the planet ring can be expressed by rotating or revolving speed. Here, the rotating speed is chosen; thus, the amplitude of excitation is $\Omega^{2} R_{0} / R$, and the excitation angular frequency is $\omega$. It should be noted that the excitation only acts on the first order component, as shown in $\widetilde{\mathbf{Q}}$ in Equation (26), after carrying out the orthogonal operation. In addition, Equation (26) represents a nonlinear dynamic system, since $\theta_{b i}$ and $\theta_{m i}$ are time dependent, as described in Section 2; then the stiffness matrix, $\mathbf{K}$, will change with respect to time, $t$. Therefore, Equation (26) will be solved by the classical numerical method.

Figure 9 shows the forced responses of the planet ring under the excitation of rotating and revolving movement at the rotating speed $\omega=1$. Here the forced responses of radial direction at different positions are illustrated. It is obvious that, although the forced responses are nonlinear, as shown in Figure 9e, the forced responses at different positions exhibit some periodic behavior with a quasi-period of rotating speed, $\omega$, as shown in Figure $9 \mathrm{a}-\mathrm{d}$. The regions with small amplitudes in Figure $9 \mathrm{a}-\mathrm{d}$ mean that the partially distributed stiffnesses are passing through this position, and the regions with large amplitude mean that the partially distributed stiffnesses are out of the position. These phenomena imply that the forced responses of the planet ring will vary with a quasi-period of its rotating speed, and the partially distributed stiffnesses will suppress its vibrations. 

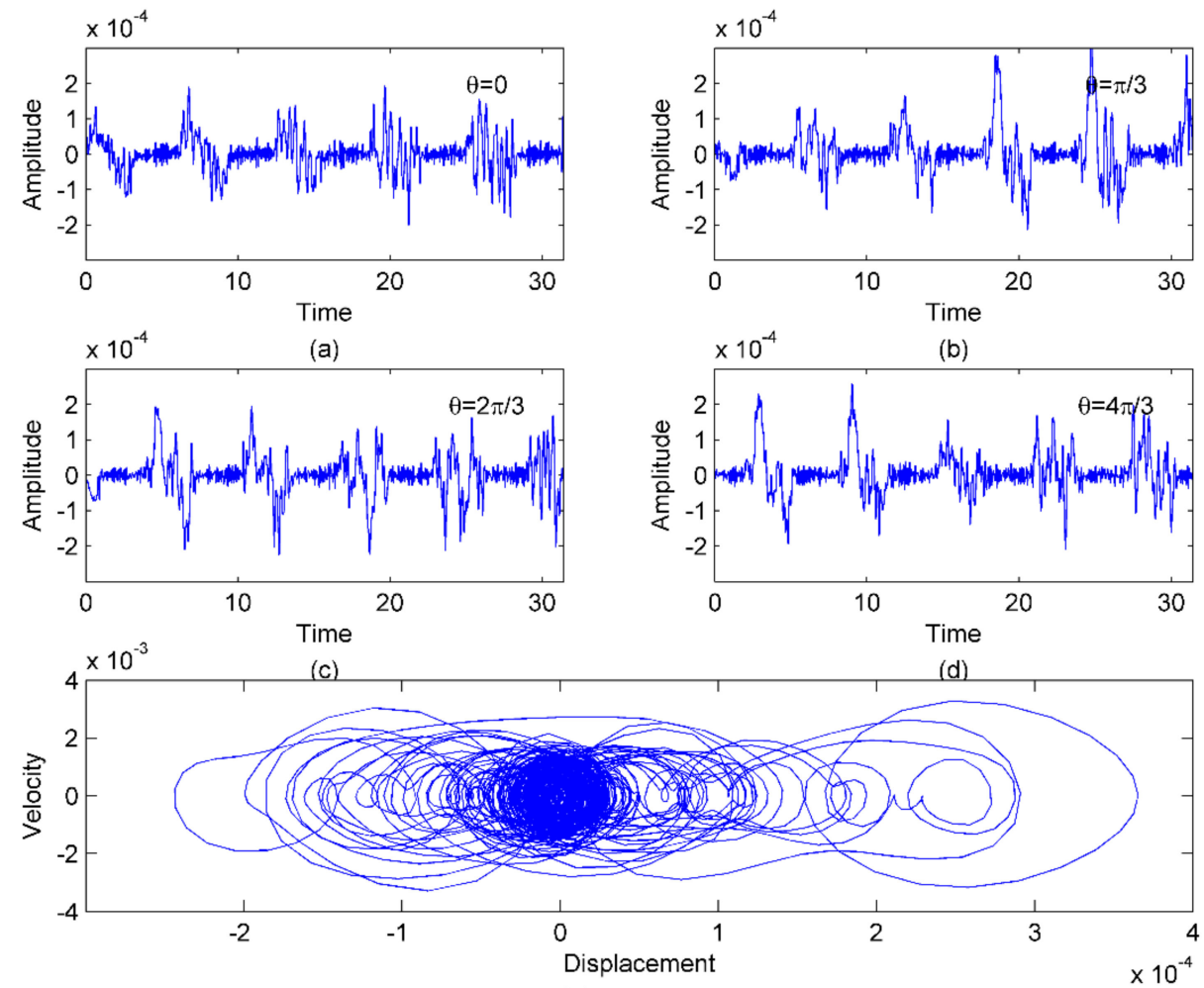

(e)

Figure 9. Forced response of the planet ring in the $\mathrm{u}$ direction at different positions, where (a) $\theta=0$, (b) $\theta=\pi / 3$, (c) $\theta=2 \pi / 3$, and (d) $\theta=4 \pi / 3$; (e) phase diagram of the position $\theta=\pi / 3$.

Figure 10 shows the forced responses of the planet ring in different moments in a period, $T$, of rotating speed at $\omega=1$. It can be found that the vibration of the planet ring is small in partially distributed stiffnesses area, and the vibration in other areas is large and irregular, that is, nonlinear. The forced responses of the planet ring almost rotate with its rotating movement.
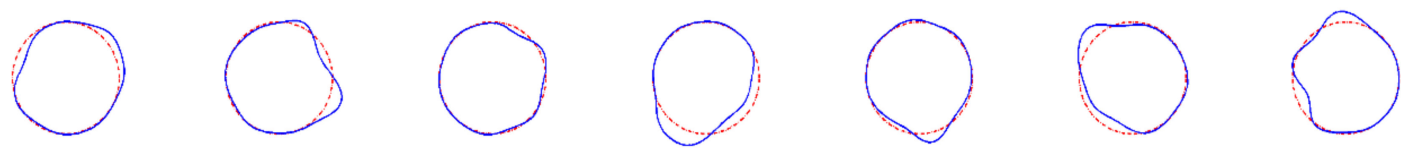

Figure 10. Forced response of the planet ring at different times in a period, $T$, of rotating speed at $\omega=1$ (dash line $=$ original position; solid line $=$ forced response).

\section{Conclusions}

This study investigated the vibration of high-speed planet rings with discrete and partially distributed stiffnesses. The governing equations were derived by Hamilton's principle based on stationary and co-rotating coordinates. The governing equation contains the revolving movement and discrete and partially distributed stiffnesses boundary conditions, where the differences with other references were given. The eigenvalue problem of the governing equations was solved by using Galerkin's method and state space matrix.

The vibration properties of a free planet ring based on parameters of a planetary gear set were investigated. The results show that there are frequency degeneracies when the rotation speed is not zero, and frequency veering occurs with the increase of rotation speed. In these regions, frequencies veer and exchange vibration mode. Each vibration mode of the free planet ring is dominated by a single nodal diameter component. When the partially distributed stiffness and discrete stiffness are acted on the planet ring, the vibration properties vary heavily. The frequencies have a distinct difference compared to 
the free planet ring. The phenomenon of frequency veering occurs with the increase of rotation speed. Each vibration mode is comprised of several nodal diameter components instead of a single component in free states. In addition, partially distributed stiffnesses will change the vibration mode heavily.

The influences of parameters on vibration properties were investigated. The results show that the distribution area and the number of partially distributed stiffnesses mainly affect the high-order frequencies. Changing the number of partially distributed stiffnesses can change the natural frequencies of the planet ring. In heavy load conditions, the natural frequencies will get lower, and in light load conditions, the natural frequencies will get higher. The discrete stiffnesses affect the natural frequencies slightly, while the partially distributed stiffnesses affect the high order natural frequencies heavily.

Finally, the forced responses of the planet ring caused by rotating and revolving movement were investigated. The results show that the forced responses are nonlinear and vary with a quasi-period of rotating speed, and the responses in the regions supported by partially distributed stiffnesses are suppressed.

Author Contributions: Conceptualization, F.Y.; methodology, F.Y.; visualization, D.W.; project administration, F.Y.; funding acquisition, F.Y. All authors have read and agreed to the published version of the manuscript.

Funding: This research was funded by the National Natural Science Foundation of China, grant number 51775309; the Scientific and Technological Development Project of Shandong Province, grant number 2019GGX104013; the State Key Laboratory of Mechanical Transmissions, Chongqing University, grant number SKLMT-KFKT-201506); and the Young Scholars Program of Shandong University.

Institutional Review Board Statement: Not applicable.

Informed Consent Statement: Not applicable.

Data Availability Statement: No new data were created or analyzed in this study. Data sharing is not applicable to this article.

Conflicts of Interest: The authors declare no conflict of interest.

\section{Appendix A. Matrices and Vectors for Equation (26)}

$$
\begin{aligned}
& {[\tilde{\mathbf{M}}]=\left[\begin{array}{ccc}
1 & 0 & 0 \\
0 & \ddots & 0 \\
0 & 0 & 1
\end{array}\right]_{4 N_{f} \times 4 N_{f}},[\overline{\mathbf{G}}]=\left[\begin{array}{cccc}
0 & 0 & \overline{\mathbf{G}}_{p \xi} & 0 \\
0 & 0 & 0 & \overline{\mathbf{G}}_{q \eta} \\
-\overline{\mathbf{G}}_{p \xi} & 0 & 0 & 0 \\
0 & -\overline{\mathbf{G}}_{q \eta} & 0 & 0
\end{array}\right]_{4 N_{f} \times 4 N_{f}}, \widetilde{\mathbf{K}}=\left[\begin{array}{cccc}
\overline{\mathbf{K}}_{p p} & \overline{\mathbf{K}}_{p q} & 0 & \overline{\mathbf{K}}_{p \eta} \\
& \overline{\mathbf{K}}_{q q} & \overline{\mathbf{K}}_{q \xi} & 0 \\
& & \overline{\mathbf{K}}_{\tilde{\xi} \xi} & 0 \\
s y m & & & \overline{\mathbf{K}}_{\eta \eta}
\end{array}\right]_{4 N_{f} \times 4 N_{f}},} \\
& \overline{\mathbf{G}}_{p \xi}(n, k)=\left\{\begin{array}{l}
-2(\Omega+\omega), n=k \\
0, n \neq k
\end{array}, \overline{\mathbf{G}}_{q \eta}=\overline{\mathbf{G}}_{p \xi},\right. \\
& \overline{\mathbf{K}}_{p p}(n, k)=\left\{\begin{array}{l}
\gamma-(\Omega+\omega)^{2}+n^{4}+N_{c}^{\prime} n^{2}+k_{b} \sum_{i=1}^{N_{b}} \cos ^{2} n \theta_{b i}, n=k \\
k_{b} \sum_{i=1}^{N_{b}} \cos k \theta_{b i} \cos n \theta_{b i}+\sum_{i=1}^{N_{b}} k_{m i} \sin \alpha \cos k \theta_{m i} \cos n \theta_{m i}, n \neq k
\end{array},\right. \\
& \overline{\mathbf{K}}_{q q}(n, k)=\left\{\begin{array}{l}
\gamma-(\Omega+\omega)^{2}+n^{4}+N_{c}^{\prime} n^{2}+k_{b} \sum_{i=1}^{N_{b}} \sin ^{2} n \theta_{b i}, n=k \\
k_{b} \sum_{i=1}^{N_{b}} \sin k \theta_{b i} \sin n \theta_{b i}+\sum_{i=1}^{N_{b}} k_{m i} \sin \alpha \sin k \theta_{m i} \sin n \theta_{m i}, n \neq k
\end{array},\right. \\
& \overline{\mathbf{K}}_{\xi \xi}(n, k)=\left\{\begin{array}{l}
(\gamma+1) n^{2}-(\Omega+\omega)^{2}+\sum_{i=1}^{N_{b}} k_{m i} \cos ^{2} n \theta_{m i}, n=k \\
\sum_{i=1}^{N_{b}} k_{m i} \cos \alpha \cos k \theta_{m i} \cos n \theta_{m i}, n \neq k
\end{array}, \overline{\mathbf{K}}_{\eta \eta}(n, k)=\left\{\begin{array}{l}
(\gamma+1) n^{2}-(\Omega+\omega)^{2}+\sum_{i=1}^{N_{b}} k_{m i} \sin ^{2} n \theta_{m i}, n=k \\
\sum_{i=1}^{N_{b}} k_{m i} \cos \alpha \sin k \theta_{m i} \sin n \theta_{m i}, n \neq k
\end{array},\right.\right.
\end{aligned}
$$




$$
\begin{aligned}
& \overline{\mathbf{K}}_{p q}(n, k)=k_{b} \sum_{i=1}^{N_{b}} \sin k \theta_{b i} \cos n \theta_{b i}+\sum_{i=1}^{N_{b}} k_{m i} \sin \alpha \sin k \theta_{m i} \cos n \theta_{m i}, \\
& \overline{\mathbf{K}}_{p q}(n, k)=k_{b} \sum_{i=1}^{N_{b}} \sin k \theta_{b i} \cos n \theta_{b i}+\sum_{i=1}^{N_{b}} k_{m i} \sin \alpha \sin k \theta_{m i} \cos n \theta_{m i}, \\
& \overline{\mathbf{K}}_{p \eta}(n, k)=\left\{\begin{array}{c}
\gamma n+n^{3}, n=k \\
0, n \neq k
\end{array}, \overline{\mathbf{K}}_{q \xi}(n, k)=\left\{\begin{array}{c}
-\left(\gamma n+n^{3}\right), n=k \\
0, n \neq k
\end{array},\right.\right. \\
& \widetilde{\mathbf{Q}}=\left\{\begin{array}{ll}
\mathbf{Q}_{c} & \mathbf{Q}_{z}
\end{array}\right\}_{4 N_{f}} \mathbf{Q}_{c}=\left\{\begin{array}{ll}
\mathbf{Q}_{p} & \mathbf{Q}_{\eta}
\end{array}\right\}_{2 N_{f}}^{\mathrm{T}} \mathbf{Q}_{s}=\left\{\begin{array}{ll}
\mathbf{Q}_{q} & \mathbf{Q}_{\xi}
\end{array}\right\}_{2 N_{f}}^{\mathrm{T}}
\end{aligned}
$$

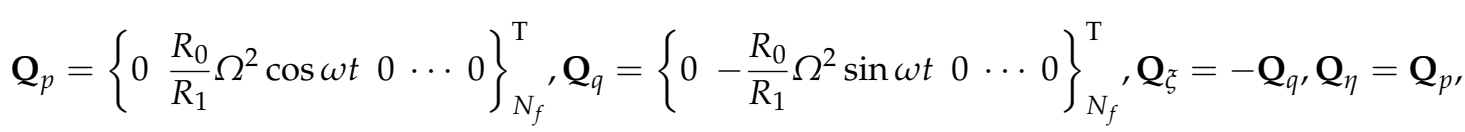

where $n, k=0,1,2, \ldots, N_{f}-1$.

\section{References}

1. Kapelevich, A.L.; Shekhtman, Y.V. Root fillet optimization of thin rim planet gears with asymmetric teeth. In Proceedings of the International Conference on Gear, Munich, Germany, 13-15 September 2017.

2. Talbert, P.B.; Gockel, R.R. Modulation of gear tooth loading due to travelling wave vibration. In Proceedings of the ASME 2003 International Design Engineering Technical Conferences and Computers and Information in Engineering Conference, Volume 4, 9th International Power Transmission and Gearing Conference, Parts A and B, Chicago, IL, USA, 2-6 September 2003.

3. Bettaieb, M.N.; Velex, P.; Ajmi, M. A static and dynamic model of geared transmission: By combining substructure and elastic foundations-application to thin rimmed gears. ASME J. Mech. Des. 2007, 129, 184-194. [CrossRef]

4. Stockton, R.J. Sun gear traveling wave vibration in a sequential planetary gearbox. In Proceedings of the ASME Design Engineering Division Conference and Exhibit on Mechanical Vibration and Noise, Cincinnati, OH, USA, 15-17 September 1985.

5. Vinayak, H.; Singh, R. Multi-body dynamics and modal analysis of compliant gear bodies. J. Sound Vib. 1998, 210, 171-214. [CrossRef]

6. Kahraman, A.; Kharazi, A.A.; Umrani, M. A deformable body dynamic analysis of planetary gears with thin rims. J. Sound Vib. 2003, 262, 752-768. [CrossRef]

7. Bert, C.W.; Chen, T.L.C. On vibration of a thick flexible ring rotating at high speed. J. Sound Vib. 1978, 61, 517-530. [CrossRef]

8. Rao, S.S.; Sundararajan, V. In-plane flexural vibrations of circular rings. ASME J. Appl. Mech. 1969, 36, 620-625. [CrossRef]

9. Detinko, F.M. Free vibration of a thick ring on multiple supports. Int. J. Eng. Sci. 1989, 27, 1429-1438. [CrossRef]

10. Bickford, W.B.; Reddy, E.S. On the in-plane vibrations of rotating rings. J. Sound Vib. 1985, 101, 13-22. [CrossRef]

11. Allaei, D.; Soedel, W.; Yang, T.Y. Natural frequencies and modes of rings that deviate from perfect axisymmetric. J. Sound Vib. 1986, 111, 9-27. [CrossRef]

12. Loy, C.T.; Lam, K.Y. Vibration of cylindrical shells with ring support. Int. J. Mech. Sci. 1997, 39, 455-471. [CrossRef]

13. Metrikine, A.V.; Tochilin, M.V. Steady-state vibrations of an elastic ring under a moving load. J. Sound Vib. 2000, 232, 511-524. [CrossRef]

14. Kim, W.; Chung, J. Free non-linear vibration of a rotating thin ring with the in-plane and out-of-plane motions. J. Sound Vib. 2002, 258, 167-178. [CrossRef]

15. Nackenhorst, U.; Brinkmeier, M. On the dynamics of rotating and rolling structures. Arch. Appl. Mech. 2008, 78, 477-488. [CrossRef]

16. Wu, X.; Parker, R.G. Vibration of rings on a general elastic foundation. J. Sound Vib. 2006, 295, 194-213. [CrossRef]

17. Wu, X.; Parker, R.G. Modal Properties of Planetary gears with an elastic continuum ring gear. ASME J. Appl. Mech. 2008, 75, 031014. [CrossRef]

18. Parker, R.G.; Wu, X. Vibration modes of planetary gears with unequally spaced planets and an elastic ring gear. J. Sound Vib. 2010, 329, 2265-2275. [CrossRef]

19. Canchi, S.V.; Parker, R.G. Parametric instability of a rotating circular ring with moving, time-varying springs. ASME J. Vib. Acoust. 2006, 128, 231-243. [CrossRef]

20. Canchi, S.V.; Parker, R.G. Parametric instability of a circular ring subjected to moving springs. J. Sound Vib. 2006, 293, 360-379. [CrossRef]

21. Cooley, C.G.; Parker, R.G. Mechanical stability of high-speed planetary gears. Int. J. Mech. Sci. 2013, 69, 59-71. [CrossRef]

22. Cooley, C.G.; Parker, R.G. Vibration of high-speed rotating rings coupled to space-fixed stiffness. J. Sound Vib. 2014, 333, 2631-2648. [CrossRef]

23. Zhao, Z.; Wang, S.; Xiu, J. Parametric vibration of an elastic structure with stationary and rotating rings subjected to traveling loads. J. Sound Vib. 2015, 358, 334-355. [CrossRef] 
24. Lu, A.; Tsouvalas, T.; Metrikine, A.V. The in-plane free vibration of an elastically supported thin ring rotating at high speeds revisited. J. Sound Vib. 2017, 402, 203-218. [CrossRef]

25. Cooley, C.G.; Parker, R.G. Eigenvalue sensitivity and veering in gyroscopic systems with application to high-speed planetary gears. Eur. J. Mech. A-Solid 2018, 67, 123-136. [CrossRef]

26. Liu, C.; Cooley, C.G.; Parker, R.G. Parametric instability of spinning elastic rings excited by fluctuating space-fixed stiffnesses. J. Sound Vib. 2017, 400, 533-549. [CrossRef]

27. Lu, T.; Tsouvalas, A.; Metrikine, A.V. A high-order model for in-plane vibrations of rotating rings on elastic foundation. J. Sound Vib. 2019, 455, 118-135. [CrossRef]

28. Harris, T.A.; Kotzalas, M.N. Advanced Concepts of Bearing Technology; CRC Press: New York, NY, USA, 2007.

29. Ginsberg, J.H.; Genin, J. Statics and Dynamics; Wiley: New York, NY, USA, 1984.

30. Meirovitch, L. A new method of solution of the eigenvalue problem for gyroscopic systems. AIAA J. 1974, 12, 1337-1342. [CrossRef]

31. Mote, C.D. Stability of circular plates subjected to moving loads. J. Frankl. Inst. 1970, 290, 329-344. [CrossRef]

32. Parker, R.G.; Sathe, P.J. Free vibration and stability of a spinning disk-spindle system. ASME J. Vib. Acoust. 1999, 121, 391-396. [CrossRef] 